\title{
Prevalence of metabolic syndrome and gender differences
}

\author{
Seerat Hussain Beigh* \& Saroj Jain
}

Department of Biochemistry, Faculty of Medicine, NIMS University, Jaipur, INDIA; Seerat Hussain Beigh - Email: seerat010@yahoo.co.in; Phone: +91-9906494497, +91-9797712356; *Corresponding author

Received June 14, 2012; Accepted June 18, 2012; Published July 06, 2012

\begin{abstract}
:
In a comparative study, involving 500 subjects with 294 males and 206 females aged 30 years and above, data were collected from NIMS (National Institute of Medical Sciences) hospital and research centre and controls from the general population whose age and sex were matched with subjects during the years 2010 - 2011. Metabolic syndrome was present both in women and men corresponding to $29 \%$ and $23 \%$ of the women's and men's sample, respectively. The prevalence was higher in women than in men. In women, elevated BMI, low HDL cholesterol, increased waist circumference and hyperglycemia were significantly larger contributors to the metabolic syndrome while in men these were hypertension and elevated triglycerides. The contribution of several metabolic components to the metabolic syndrome is different in men and women. This might contribute to gender specific differences in the relative risk of metabolic complications such as insulin resistance.
\end{abstract}

Keywords: Prevalence, Metabolic Syndrome, Gender differences, Insulin resistance, BMI

\section{Background:}

The metabolic syndrome is characterized by a cluster of several metabolic and cardiovascular risk factors such as abdominal obesity, insulin resistance, atherogenic dyslipidemia, and hypertension [1]. Although there are different definitions of the metabolic syndrome, the uniform pathophysiology of this syndrome is insulin resistance [2]. The importance of the metabolic syndrome lies largely in that the cardiovascular risk factors increase the risk of cardiovascular disease by 2-fold and the risk of developing type 2 diabetes mellitus by 3 -fold [2]. The etiology, prevention and treatment of the metabolic syndrome currently are the focus of intense research activities. The combination of abdominal obesity, hypertension, dyslipidemia, hyperglycemia and insulin resistance or type 2 diabetes mellitus defines the metabolic syndrome [3].

It has been reported that women develop cardiovascular disease (CVD) at an older age compared to men. Gender differences in this syndrome may contribute to differences in CVD [4,5]. The contributions of different components of the metabolic syndrome differ between genders and in different countries [4]. The underlying risk factors appear to be abdominal obesity and insulin resistance, other associated conditions are physical inactivity, ageing and polycystic ovarian syndrome [6]. Elevated blood pressure (BP) is also associated with insulin resistance or hyperinsulinemia [7]. Similarly, smokers and heavy alcohol users often have Metabolic Syndrome [7]. Published reports differ in the gender distribution of the metabolic syndrome $[5,6]$. Some researchers report a higher incidence of the metabolic syndrome in men [5, 6] than women, the reverse is the case in some other reports [7, 8]. This study aims to determine gender specific prevalence as well as the components of the metabolic syndrome within the jurisdiction of Jaipur city of Rajasthan State.

\section{Methodology:}

The study was undertaken with participants including 294 men and 206 women, aged 30 years and above. Convenient hospitalized patients with the diagnosis of acute myocardial infarction were included, while those with the history of steroids, chronic renal or hepatic disease were excluded. Healthy age and sex matched controls from the general 
population were also taken. Controls with previous history of myocardial infarction, hypertension and diabetes were excluded. The facilities of NIMS hospital and research centre were used for different aspects of the study.

The study participants underwent a clinical assessment after obtaining an informed written consent. A detailed medical history with the help of questionnaire and a clinical examination including body mass index, waist circumference and blood pressure were recorded. Participants who were on anti hypertensive or anti diabetic drugs were considered as hypertensives and diabetics. Dyslipidemia was considered in those already on lipid lowering drugs. Laboratory assessment included obtaining fasting venous blood samples for the determination of the lipid profile, and blood glucose. Ethical approval was obtained from the Ethical committee of hospitals. The presence of the metabolic syndrome was determined using the National Cholesterol Education Program Adult Treatment Panel (NCEP ATP III) definition Asian modified. The presence of three or more of any of the following was pointer to the MetS 1) Waist circumference (WC) greater than $90 \mathrm{~cm}$ in men and 80 $\mathrm{cm}$ in women; 2) Serum triglycerides (TG) level of at least 150 mg/dL (1.69 mmol/L); 3) High-density lipoprotein cholesterol (HDL-C) level of less than $40 \mathrm{mg} / \mathrm{dL}(1.04 \mathrm{mmol} / \mathrm{L})$ in men and $50 \mathrm{mg} / \mathrm{dL}(1.29 \mathrm{mmol} / \mathrm{L})$ in women; 4) Blood pressure of at least 130/85 mm Hg; 5) Serum glucose level of at least 110 $\mathrm{mg} / \mathrm{dL}(6.05 \mathrm{mmol} / \mathrm{L})$. We compared between the gender differences for age, obesity, waist circumference, blood pressure, triglycerides, fasting blood sugar and HDL-C by using Chi square test. Difference between males and females regarding the frequency of metabolic syndrome were also assessed. Statistical significance of P-value was taken as $<0.05$. The data were analyzed using statistical tools.

\section{Result:}

The mean age of the study subjects was 55 - 56_+ (S.D of 15.22) years. The most important metabolic syndrome parameters in the study subjects were central obesity (increased waist circumference $29 \%$ ) elevated triglycerides 33\%), hypertension $38 \%$, low HDL (36\%) and hyperglycemia in (29\%). Most of the women had elevated BMI $(50 \%)$ and were postmenopausal. As compared to men almost all were house wives with poor physical activity and $13 \%$ of literacy which were also significant statistically Sign.

Gender differences were seen as significant statistically regarding HDL cholesterol, waist circumference, and fasting glucose levels while they were similar regarding triglyceride and BP. Using the standard cutoff values of the individual components for metabolic syndrome the distribution was as follow: the low HDL cholesterol was seen in $25 \%$ of females as compared to $34 \%$ males (P-value $<0.0001$ ). $42 \%$ of males had waist circumference $>/=90 \mathrm{~cm}$, while $63 \%$ females had a waist circumference $>/=80 \mathrm{cms}$ (P-value $<0.002)$. Similarly hyperglycemia also significantly differed in males as compared to females i.e. $25 \%$ vs $42 \%$ ( $\mathrm{P}$ value $<0.01$ ) respectively. The difference among males and females regarding BMI was only marginal ( $p$-value 0.08). Metabolic syndrome according to ATPIII criteria i.e. those who had three or more risk factors, was found in $128(25.6 \%)$ of study participants. Table 1 (see supplementary material) shows that in both women and men a cluster corresponding to the metabolic syndrome represented
$29 \%$ and $23 \%$ of the women's and men's samples, respectively the statistically results were analysed to be significant which was also statistically significant.

\section{Discussion:}

Approximately 47 million Americans meet the diagnostic criteria of metabolic syndrome, corresponding to about $40 \%$ of the adult population according to census data of 2000 [9]. Somewhat similar results were seen in our study; 25.6 percent i.e. 128 of study participants had metabolic syndrome. The components of the metabolic syndrome vary in their rates of occurrence. The Seychelles study reported high blood pressure and adiposity as the metabolic syndrome defining criteria that occurred most commonly irrespective of the metabolic syndrome definitions used Ogbera. A however reported central obesity and reduced HDL-cholesterol as the prevalent components of the metabolic syndrome [1]. The common components of metabolic syndrome included central obesity and dyslipidemia in our study. This finding is not surprising as it has been seen that central obesity plays a central role in the development of the metabolic syndrome and appears to precede the appearance of the other metabolic syndrome components [4].

The gender differences in prevalence of metabolic syndrome have been found in several studies. It might be due to different cut-off points set as criteria for metabolic syndrome like WC and HDL-C [4]. The results of gender females dominance was seen in our data similar to the Arkhangel study done in Russia Korea and China [8, 10-12]. While it is comparable to the prevalence data in Frinks cohort [13] study, and the studies conducted in Pakistan [14, 15]. It was worrisome that the increase in prevalence of the metabolic syndrome was higher in women than in men. This is mainly driven by the constant rise in obesity in women, with presently 2 million more women than men being affected in the United States ${ }^{4}$ as well as in the under developing countries including south east asian countries $[6,14]$.

Different studies have been undertaken to characterize the major components of the metabolic syndrome in women and men in different populations. In a large community based crosssectional survey in Mauritius, non-diabetic women had significantly higher body mass indices and 2-h glucose levels than non-diabetic men [16]. Investigations performed in the French Monica cohorts to separate the contributions of different factors to the metabolic syndrome showed a significantly elevated body weight, waist girth, and low HDL cholesterol in women than in men [17]. Similar findings were seen in our study i.e. increased waist girth (central obesity), and low HDL were present more in women as compared to men while blood pressure and triglycerides levels were same in both the gender. Abdominal fat tissue is a major source of free fatty acids and cytokines for the liver, favoring the early development of insulin resistance, dyslipidemia, and high blood pressure. Thus, women need a higher degree of adiposity to achieve the same metabolic disturbances. This might be explained by the more favorable fat distribution in women [4]. Most of the women in our study were postmenopausal with android distribution of fat. In a US study, metabolic syndrome was associated with physical inactivity in overweight men and in normal weight and overweight women, suggesting a high protective value of 
physical exercise in women. Similarly the women were having more physical inactivity as compared to men in our study. Other protective life styles are also effective, but are unfortunately heavily underused - such as a high cereal fiber intake, non-smoking and de-addiction of alcohol.

\section{Conclusion:}

The pathophysiology of the metabolic syndrome and its contribution to the relative risk of cardiovascular events and heart failure show gender differences that has immense potential relevance for prevention, diagnostics, and therapy of the metabolic syndrome in all age-groups and all geographical regions.

\section{References:}

[1] Deedwania PC et al. J Assoc Physicians India. 2006 54: 797 [PMID: 17214277]

[2] Reaven G, Circulation. 2002 106: 286 [PMID: 12119239]

[3] Alberti KG et al. Circulation. 2009 120: 1640 [PMID: 19805654]

[4] Regitz-Zagrosek V et el. Clin Res Cardiol. 2006 95: 136 [PMID: 16598526]
[5] Njelekela MA et al. BMC Cardiovasc Disord. 2009 9: 30 [PMID: 19615066]

[6] Fezeu L et al. Atherosclerosis. 2007 193: 70 [PMID: 17011567]

[7] Ahonen $\mathrm{T}$ et al. Mediators Inflamm. 2009 1: 6 [PMID: 19707530]

[8] He Y et al. Diabetes Care. 2006 47: 1588 [PMID: 17468350]

[9] Ford ES, Diabetes Care. 2005 28: 2745 [PMID: 16249550]

[10] Sidorenkov O et al. BMC Public Health. 2010 10: 23 [PMID: 20085638]

[11] Park HS et al. Int J Epidemiol. 2004 33: 328 [PMID: 15082635]

[12] Park HS et al. Diabetes Care. 2006 29: 933 [PMID: 16567843]

[13] Ilanne-Parikka P et al. Diabetes Care. 2004 27: 2135 [PMID; 15333474]

[14] Jahan F et al. J Coll Physicians Surg Pak. 2007 17: 32 [PMID: 17204217]

[15] Ashraf SMS et al. Pak J Med Sci. 2006 22: 295

[16] Williams JW et al. Diabet Med. 2003 20: 915 [PMID: 14632717]

[17] Dallongeville J et al. Ann Nutr Metab. 2004 48: 43 [PMID: 14646340]

Edited by $\mathbf{P}$ Kangueane

Citation: Beigh \& Jain, Bioinformation 8(13): 613-616 (2012)

License statement: This is an open-access article, which permits unrestricted use, distribution, and reproduction in any medium, for non-commercial purposes, provided the original author and source are credited 


\section{BIOINFORMATION}

\section{Supplementary material:}

Table 1: Prevalence of gender-wise metabolic syndrome.

\begin{tabular}{lll}
\hline Metabolic Syndrome $\quad(\mathbf{N}=\mathbf{5 0 0})$ & Women $\mathbf{( \% )}$ & Men (\%) \\
\hline Metabolic Syndrome present & $60(29.13 \%)$ & $68(23.13 \%)$ \\
Metabolic Syndrome absent & $146(70.87 \%)$ & $226(76.87 \%)$ \\
\hline
\end{tabular}

